\title{
La Conferencia de Chapultepec (1945): El nacionalismo económico latinoamericano frente a la política librecambista de Estados Unidos
}

\section{The Conference of Chapultepec (1945): Latin American Economic Nationalism versus the Free Market Policies of the United States}

\author{
José Galindo \\ Universidad Veracruzana, Veracruz, México, email: jgalindor@yahoo.com
}

Resumen: El presente artículo busca ilustrar el cambio de modelo económico en América Latina a partir de la crisis de 1929 y la transición del modelo enfocado "al exterior" al "mercado interno", utilizando, principalmente, el caso de México. Asimismo, se explicarán los efectos de la segunda guerra mundial en las principales economías latinoamericanas y el surgimiento de políticas explícitas de industrialización en dichos países. Basándonos en el análisis de fuentes primarias y secundarias, se examinará la llamada Conferencia de Chapultepec (1945) con énfasis en la estructura del debate y contraste en las posturas de los países latinoamericanos, interesados en mantener y profundizar el nacionalismo económico y sus procesos de industrialización, que lograron moderar las propuestas económicas de corte librecambista impulsadas por Estados Unidos.

Palabras clave: Conferencia de Chapultepec; industrialización; mercado interno; liberalismo; CEPAL.

Abstract: The present article seeks to illustrate the change of economic model in Latin America from the crisis of 1929 and the transition from the model focused "outwards" to the "internal market", using mainly the case of Mexico. It will also explain the effects of the Second World War on the major Latin American economies and the emergence of explicit industrialization policies in these countries. Based on the analysis of primary and secondary sources, the so-called Chapultepec Conference (1945) will be examined, with an emphasis on the structure of debate and contrast in the positions of Latin American countries, interested in maintaining and expanding economic nationalism and its processes of industrialization, which managed to moderate the freetrade economic proposals promoted by the United States

Key words: Conference of Chapultepec; industrialization; domestic market; liberalism; ECLAC.

Fecha de recibido: 17 de junio de 2016. Fecha de aceptado: 31 de octubre de 2016.

Am. Lat. Hist. Econ., may.-ago., 2017, pp. 35-58 | DOI: 10.18232/alhe.v24i2.800 


\section{INTRODUCCIÓN}

$\mathrm{E}$ l objetivo de este trabajo es profundizar en los efectos que tuvieron las propuestas realizadas por Estados Unidos en el marco de la Conferencia Interamericana sobre Problemas de la Guerra y de la Paz (Conferencia de Chapultepec), celebrada en la ciudad de México en 1945, y la política económica llevada a cabo a partir de tal suceso por los países latinoamericanos, particularmente México.

Desde antes del inicio de la segunda guerra mundial, gran parte de los países de América Latina ya estaban inmersos en un proceso de industrialización, producto de la sustitución de importaciones. Durante el periodo de guerra, la dinámica de las economías latinoamericanas, en general, sufrió algunos cambios que principalmente incentivaron una mayor industrialización; en parte por la escasez de productos antes importados de países del centro. ${ }^{1}$ En este contexto, y antes de que terminara el conflicto bélico, se llevó a cabo la Conferencia de Chapultepec, en la que además de tocar temas como la solidaridad americana y la creación de una organización regional permanente, se propusieron algunas políticas a los Estados americanos para su futuro progreso económico. Como se verá en el desarrollo del presente trabajo, las propuestas económicas de Estados Unidos contrastaron en algunos puntos clave con la posición general de los países de latinoamérica, manteniéndose esta última como la directriz de la política económica que se implementó en la mayoría de ellos, en contraposición a los deseos del, entonces ya, país más poderoso del mundo.

Mi hipótesis establece que en el marco de la Conferencia de Chapultepec los países latinoamericanos lograron moderar el avance de las políticas económicas de Estados Unidos, en especial la denominada Carta Económica de las Américas, que originalmente era una propuesta en línea con la aplicación de políticas liberales y de plena apertura comercial. Ello permitió que en años posteriores fuera posible dar cauce a las políticas de los países de América Latina para continuar y profundizar el proceso de industrialización basado en un modelo económico nacionalista.

Para desarrollar esta hipótesis, primeramente introduciré el proceso de cambio del modelo económico de América Latina a partir de la crisis de 1929. La transición de modelo de crecimiento enfocado "al exterior" a uno orientado hacia el mercado interno y basado en el impulso a la industria que, en un principio, no fue resultado de políticas explícitas de in-

\footnotetext{
${ }^{1}$ Esta expresión hace referencia a la terminología empleada por los cepalistas (más adelante hablaremos de la CEPAL) para denominar a los países más industrializados, que se beneficiaban de la importación de materias primas, principalmente, proveniente de los países cuya economía dependía en gran medida de la exportación de estos productos: periferia.
} 
dustrialización. ${ }^{2}$ En segundo lugar, hablaré sobre los efectos de la segunda guerra mundial en las economías latinoamericanas y el surgimiento de las políticas explícitas de desarrollo industrial para la región durante los primeros años de la década de 1940. En tercer lugar, analizaré la Conferencia de Chapultepec, a partir del caso de México, para ejemplificar la postura de los países latinoamericanos en ciertos temas, como el comercio internacional y nacionalismo económico, y los contrastaré con las propuestas de Estados Unidos. Al final de esta sección, también haré referencia a otras conferencias interamericanas, como la de La Habana de 1947, donde se confirma la oposición de algunos países de América Latina contra las propuestas estadunidenses. Finalmente, mencionaré la labor paralela que realizó la Comisión Económica para América Latina (en adelante CEPAL) para justificar teóricamente la continuación de la política de industrialización basada en la sustitución de importaciones en la región, y así evitar los efectos negativos de permanecer dentro de un modelo de exportación de materias primas. $\mathrm{Al}$ final realizaré una sección de conclusiones. ${ }^{3}$

\section{EL PROCESO DE INDUSTRIALIZACIÓN \\ Y EL CAMBIO DE MODELO ECONÓMICO}

Para poder hablar de cambio de modelo económico es importante comenzar por definir este concepto. Un modelo de crecimiento económico es el proceso de acumulación de capital que se lleva a cabo dentro de los límites que impone una estructura económica determinada (Millán, 1998, p. 11). Estas estructuras están compuestas por la dotación de recursos de una economía y por el marco de incentivos de los agentes económicos, es decir, el marco que determina hacia dónde fluyen las preferencias de los agentes económicos. ${ }^{4}$ Como las estructuras económicas están relacionadas con las instituciones y organismos que operan dentro de una economía, así como con las preferencias individuales, estas crean una poderosa inercia de continuidad del patrón que sigue la economía, lo que permite que los modelos de crecimiento sean relativamente estables. Es importante mencionar que los modelos de crecimiento tienen dos elementos básicos: el

${ }^{2}$ Esto no quiere decir que los gobiernos no tuvieran algún tipo de políticas que incentivaran el desarrollo industrial. Sin embargo, la idea de que la industria se convirtiera en el motor del desarrollo económico emergió después.

${ }^{3}$ Es importante mencionar que el país base de toda esta investigación es México. Sin embargo, como la historia económica de América Latina comparte muchas características con las de este país, se hará referencia, en la medida de lo posible, a otros ejemplos regionales.

${ }^{4}$ El marco de incentivos, a su vez, está determinado por el marco institucional. Para un análisis detallado sobre el significado e implicaciones del marco institucional, véase North (1993). 
sector económico que impulsa a la economía (motor de la economía), es decir, aquel sector que crece más rápido que los demás, y la orientación de la economía, que puede ser hacia el mercado interno o externo.

Con esta información podemos decir que, entre el último cuarto del siglo XIX y primer tercio del XX, la mayoría de las economías latinoamericanas estuvieron inmersas en un "modelo de crecimiento hacia el exterior". Este modelo tenía como motor de la economía al sector de bienes primarios y su orientación era el mercado externo. En el caso particular de México, el modelo de crecimiento al exterior se considera que surge a partir de 1876, con la llegada de Porfirio Díaz al poder, y concluye en 1929, con el inicio de la Gran Depresión. Sin embargo, más que el desarrollo de este modelo, a nosotros nos interesa explicar cómo se dio la transición entre este modelo y el "modelo de crecimiento hacia el interior", cuyo motor de la economía se basó en el sector industrial y la orientación fue hacia el mercado interno. ${ }^{5}$

Como en otras naciones latinoamericanas, la economía mexicana se pudo recuperar relativamente rápido de los efectos negativos de la Gran Depresión. Para 1932, el ciclo económico de México había alcanzado su punto más bajo y en ese mismo año comenzó la recuperación económica del país. Enrique Cárdenas, quien ha estudiado detalladamente este tema, afirma que existieron dos factores fundamentales que permitieron esta recuperación: un incremento temprano en el valor de las exportaciones mexicanas y una serie de políticas monetarias y fiscales expansionistas (Cárdenas, Ocampo y Thorp, 2000, p. 202).

El aumento en el valor de las exportaciones fue particularmente importante en el sector minero y petrolero. Para 1934, estas sobrepasaron su valor en dólares de ventas al exterior de 1929, contribuyendo substancialmente a la recuperación temprana del total de las exportaciones. De los 82000000 de dólares de incremento de las exportaciones de 1932 a 1934, 77\% vino del oro, plata y petróleo (Cárdenas, 1982, pp. 319-322). Consecuentemente, esta posición privilegiada de México de tener petróleo y plata, le permitió, además de reactivar su mercado interno, hacerse de divisas para importar materias primas para la rápida recuperación de

\footnotetext{
${ }^{5}$ En realidad, el caso mexicano es un caso excepcional en cuanto al modelo de crecimiento hacia afuera. Es un hecho que las exportaciones de bienes primarios tuvieron un dinamismo excepcional en este periodo. Sin embargo, el desarrollo de las manufacturas no vinculadas con el sector exportador tuvieron también un gran desarrollo en este periodo. Es por muchos aceptado que el desarrollo de las manufacturas se benefició de la entrada de divisas producto de las exportaciones; las que permitieron la importación de bienes de producción. Sin embargo, muchas de estas importaciones se realizaron de manera totalmente ajena al sector exportador, utilizando divisas producto de créditos obtenidos directamente de Europa, como fue el caso de muchas de las importaciones realizadas por los inmigrantes franceses. Estos inmigrantes fueron los principales productores de manufacturas en este periodo.
} 
la producción industrial. ${ }^{6}$ Varias repúblicas latinoamericanas también gozaron de una importante recuperación de su sector exportador en los años que siguieron a la crisis de 1929. Bulmer-Thomas lo atribuye, en parte, al compromiso de las autoridades por preservar al sector exportador, así como a la "lotería de productos" que benefició a algunos países y perjudicó a otros (Bulmer-Thomas, 1994, pp. 219-220). Esto último se dio, por ejemplo, en el caso de Chile, quien exportaba principalmente cobre, cuyo valor no sobrepasó al de 1929 sino hasta los años cuarenta. Otros ejemplos menos drásticos fueron los casos de Colombia y Brasil, en donde su principal producto de exportación fue el café, cuyo precio se colapsó después de 1929 (Bulmer-Thomas, 1994, p. 221).

El otro factor que permitió la rápida recuperación de las economías latinoamericanas, y que fue importante en el cambio estructural de las mismas, fue la política cambiaria adoptada. La mayoría de los países latinoamericanos no pudo mantener la paridad cambiaria ante la crisis. Aquellos que intentaron defender su tipo de cambio, como Colombia, vieron caer sus reservas en oro y divisas rápidamente (Thorp y Londoño, 2000, pp. 84-85). En el caso mexicano, el secretario de Hacienda, Alberto Pani, dejó flotar libremente el tipo de cambio en marzo de 1932, por lo que para noviembre de 1933, cuando se decide volver a fijar la paridad del peso, la devaluación respecto a 1929 había sido casi de 70\%. Esto evidentemente cambió los precios relativos entre las importaciones de productos industriales respecto a los productos de origen nacional, que competían con las primeras, lo que fomentó la sustitución de importaciones. En relación con la política monetaria, las medidas tomadas por el secretario Pani fueron esencialmente expansivas. Se volvió a la acuñación de moneda de plata y el pago a los empleados gubernamentales se hizo con billetes emitidos por el banco central. También el Banco de México redujo el requerimiento de reservas a los bancos comerciales. Estas medidas tuvieron un fuerte efecto de expansión sobre la demanda agregada. México no fue el único país de la región en adoptar políticas económicas flexibles. Bulmer-Thomas (1997), en un estudio general de las economías de la región, menciona que "el renacimiento del sector exportador, junto con políticas monetarias y fiscales poco estrictas, produjeron una expansión de la demanda interna nominal final" (p. 35).

${ }^{6}$ Es interesante hacer notar que los precios de minerales industriales como cobre, zinc y plomo, que habían sido los principales minerales que México había exportado durante el periodo de "crecimiento hacia fuera", tuvieron una lenta recuperación. Sin embargo, México fue afortunado de contar con otros productos que permitieron que parte de la recuperación económica del país fuera vía el mercado externo.

${ }^{7}$ Este término fue inventado por Carlos Díaz Alejandro al referirse a la variedad de productos primarios exportables de las economías periféricas. 
Las políticas expansivas y la recuperación de las exportaciones mexicanas permitieron la reactivación del sector industrial, no obstante hubo otros factores importantes que generaron la transferencia de recursos del sector tradicional al sector manufacturero de la economía. Hubo un aumento de 86\% de los términos de intercambio domésticos entre 1929 y 1940 (Cárdenas, Ocampo y Thorp, 2000, p. 204); es decir, de los precios urbanos relativos a los rurales. Esto desincentivó la inversión en el campo. Además, en México, el programa de reforma agraria del presidente Lázaro Cárdenas generó elementos de incertidumbre para la inversión en el campo, lo que también promovió un aumento de inversión en el sector industrial. Un tercer factor que influyó en la transferencia de recursos hacia el sector industrial fue que durante los años treinta hubo una serie de inversiones públicas importantes. En particular, la construcción de la red carretera se aceleró, lo que permitió la fusión de los centros industriales y urbanos. Esto redujo los costos de transporte y amplió el mercado interno, lo que provocó que la productividad en general aumentara. Las industrias de cemento y acero también fueron estimuladas por el aumento en las obras de infraestructura de la segunda mitad de la década de los treinta (Haber, 1989, p. 177). El crecimiento de los beneficios en el sector moderno se reflejó en un fuerte proceso de acumulación de capital, lo que permitió que la industria mexicana creciera en promedio a una tasa de $6.1 \%$ en términos reales entre 1932 y 1940 (Cárdenas, Ocampo y Thorp, 2000, p. 204). Esto llevó al sector industrial a convertirse en el motor de la economía por primera vez. ${ }^{8}$

La discusión previa ha mostrado que una importante consecuencia de la Gran Depresión fue la aceleración del proceso de sustitución de importaciones y la industrialización doméstica por medio del cambio en los precios relativos. Este resultado también fue influido por una política macroeconómica expansiva y el mantenimiento de una alta demanda agregada por el resto de los años treinta. Este último factor está relacionado con el segundo efecto más importante de la Gran Depresión: el más amplio papel del Estado en el manejo de la política macroeconómica. Los Estados latinoamericanos pudieron por primera vez implementar una política monetaria autónoma, en parte por haber dejado el patrón oro. Es decir, no tuvieron que mantener la paridad cambiaria, lo que a su vez les permitió tener mayor control sobre la oferta monetaria. Asimismo, el establecimiento de bancos centrales bajo el control del Estado permitió regular

\footnotetext{
${ }^{8}$ La contribución de la sustitución de importaciones en el crecimiento de la oferta industrial en los años treinta, es comparable con la de Brasil, en donde la industrialización en ese periodo fue también en gran parte producto del reemplazo de productos antes importados. En el caso brasileño, el crecimiento de sectores manufactureros como cemento, metalurgia, papel, mobiliario y piel, fue impresionante. Véase Fishlow (1972).
} 
la circulación monetaria, las tasas de interés y el tipo de cambio. Por otro lado, los déficits fiscales que aparecieron durante los años treinta pudieron ser financiados con el impuesto inflacionario, lo que permitió la acuñación de nueva moneda.

Dicho crecimiento del papel del Estado en la economía fue reafirmado durante la segunda guerra mundial. En México, hasta la expropiación petrolera de marzo de 1938, el gobierno de Cárdenas se concentró en aspectos sociales. El reparto agrario, educación, salud, indigenismo, cultura y la política sindical fueron las áreas en las cuales se concentró la actividad gubernamental. Pero a partir de la expropiación petrolera, Cárdenas empezó a manifestar una marcada preocupación por el desarrollo industrial. Ya había hecho algunos avances en este aspecto, pero fundando instituciones más que defendiendo políticas en la materia. En 1936 creó los Almacenes Nacionales de Depósito, en 1937 el Banco Nacional de Comercio Exterior, El Banco Nacional Obrero de Fomento Industrial y la Comisión Federal de Electricidad (Medina, 1995, p. 129), además reformó la Ley de Seguros. Aunque Cárdenas dio el primer paso de fomento a la industria en 1939, al establecer algunas medidas como exenciones fiscales por cinco años a empresas con actividades industriales nuevas, su sucesor, Ávila Camacho, definió la política industrial para aprovechar las oportunidades que trajo la guerra. ${ }^{9}$

La segunda guerra mundial permitió la continuación del proceso de diversificación económica que experimentaba América Latina. La región se convirtió en un importante proveedor de materias primas hacia los países en guerra, principalmente para Estados Unidos y Reino Unido, y al mismo tiempo pudo continuar con el proceso de sustitución de importaciones gracias a la escasez de productos importados que se presentó en ese periodo. Durante el conflicto bélico tanto la demanda interna como la externa aumentaron de forma considerable. Esto permitió un crecimiento industrial importante en la región. Las tasas de crecimiento industrial anual de Chile, México ${ }^{10}$ y Venezuela estuvieron por arriba de 9\% (Knight, 1991, p. 309). En sí, el crecimiento industrial de América Latina fue en promedio de 5.7\%, entre 1939 y 1945 (Bulmer-Thomas, 1994, p. 244). Por su parte, el Estado mexicano llevó a cabo una política de gasto público que favoreció a las inversiones en infraestructura, principalmente comunicaciones

\footnotetext{
${ }^{9}$ Aunque Cárdenas desarrolló políticas de corte radical y tuvo severos conflictos con algunos grupos de empresarios, como el Grupo Monterrey, no obstaculizó el desarrollo de la industria. De hecho, entre 1935 y 1939, más de 6000 industrias fueron establecidas en el territorio nacional. Fue un periodo en que nuevas industrias, diferentes a las tradicionales, propiedad de la elite económica tradicional que se mantenían desde el porfiriato, fueron creadas. Para un estudio detallado sobre la elite económica tradicional porfiriana, véase Galindo (2012).

${ }^{10} \mathrm{El}$ crecimiento industrial en México se dio sobre todo en textiles, comida, químicos y metales.
} 
y energía eléctrica, y una política fiscal de baja imposición y exenciones fiscales, que continuó con la promoción de nuevas industrias, así como de aquellas donde la oferta interna no satisfacía la demanda. Además, México contaba con capacidad industrial ociosa que pudo ser utilizada durante los años de guerra. En el caso mexicano, hubo además una relación económica muy estrecha con Estados Unidos. En 1940, el 90\% de los productos de exportación nacionales fueron adquiridos por el vecino del norte; igualmente, México importó el 90\% de sus productos de Estados Unidos en 1944 (Knight, 1991, p. 305). Esta dinámica económica con Estados Unidos también favoreció al proyecto gubernamental de industrialización, ya que este proveyó un mercado grande para los productos mexicanos y una fuente de bienes de capital e inversión.

Los propósitos de industrialización también se reflejaron en cambios institucionales. Se hicieron reformas a la Ley Orgánica del Banco de México, que permitieron que en 1942 esta institución comenzara con una política de canalización de créditos a la industria. Se dispuso que los bancos asociados quedaran obligados a integrar su cartera cuando menos con $60 \%$ de créditos a la producción. Nacional Financiera también se reorganizó, su función central sería el financiamiento de la industria del país (Knight, 1991, p. 309).

Por otra parte, la segunda guerra mundial dejó experiencias y convicciones en la mentalidad de la clase gobernante mexicana. De todas ellas, la certidumbre que la industrialización era la vía adecuada para alentar el crecimiento económico, crear empleos y mejorar la distribución del ingreso nacional, que sin duda fue la más importante. ${ }^{11}$ México salió de la guerra con un sector industrial acostumbrado a trabajar a plena capacidad y con cierta experiencia del mercado estadunidense y latinoamericano. De ahí, la convicción de alentar el crecimiento de la demanda interna y ampliar los mercados externos. Además, durante los años de guerra, la intervención directa del gobierno en la actividad económica, como vimos, fue de suma importancia. Por su parte, para 1945 se dejaba sentir de nuevo la competencia de la industria estadunidense que, con menores cargas relacionadas con el apoyo al esfuerzo bélico, reconvirtió sus plantas de producción de guerra a plantas de producción de paz. De manera simultánea, la demanda estadunidense de materias primas empezó a disminuir en 1945; tal fue el caso de la minería. Otro factor, no menos importante, fue la alta tasa de inflación, que para 1945 se unió a las demás causas para hacer menos competitivos los productos mexicanos en el mercado estadunidense. La inflación no sólo afectó a México, "en un número de los más

\footnotetext{
${ }^{11}$ Dos estudios que profundizan en el tema de la transformación de las mentalidades a nivel elites política y económica en este periodo son los de Mosk (1950) y Tannenbaum (1956).
} 
importantes países latinoamericanos [...] la inflación de los años de guerra probaría ser un cáncer que nunca fue extirpado, a pesar de los repetidos intentos, en el periodo de posguerra" (Bulmer-Thomas, 1994, p. 267). En promedio, la inflación regional fue de 13\% entre 1939 y 1945 (BulmerThomas, 1994, p. 250).

\section{la Conferencia de Chapultepec}

En estas condiciones se llevó acabo la Conferencia Interamericana sobre los Problemas de la Guerra y de la Paz, mejor conocida como Conferencia de Chapultepec. Al término de la segunda guerra mundial, la política internacional y el sistema de relaciones entre naciones debían reorganizarse por completo. Lo anterior, como consecuencia del efecto de la crisis y guerra. El bipolarismo que ya empezaba a perfilarse entre la URSS y Estados Unidos obligó a una alineación particular entre las naciones, y Latinoamérica acabó por formar parte de la esfera estadunidense.

Estados Unidos había comenzado a asumir su posición de potencia mundial al dictar los lineamientos que los gobiernos latinoamericanos debían seguir en el periodo de la posguerra. Washington había presionado a los Estados de la región a que rompieran relaciones diplomáticas con los países del Eje, inclusive a través de la imposición de medidas coercitivas contra los Estados que se rehusaran a abandonar su postura de neutralidad. Ejemplo de ello fue el caso argentino, que de 1942 a 1945 fue sujeto a la marginalización política y económica de la mayor parte de los países de la región, hasta que decidió alinearse a la posición hemisférica impulsada por Estados Unidos (Morgenfeld, 2010, pp. 194-196).

Otro factor influyente, en el contexto de la Conferencia de Chapultepec, fue la expectativa de los países latinoamericanos de recibir por parte de Estados Unidos ayuda económica para detonar y fortalecer sus procesos de industrialización. Se esperaba que al término de la guerra, Washington implementaría una suerte de "Plan Marshall" para Latinoamérica, pero con el tiempo fue claro que Estados Unidos tenía otros planes para la región. No obstante, dichas expectativas flexibilizaron la postura de los países latinoamericanos hacia ciertas políticas impulsadas por Washington. Ello logró, por ejemplo, que los países de la región que aún no lo habían hecho, declararan la guerra contra el Eje antes o tiempo después de la Conferencia de Chapultepec (Morgenfeld, 2010, p. 204).

En este periodo, Estados Unidos también logró unificar a los países del continente para establecer un bloque regional de cara a la Conferencia de San Francisco, donde finalmente se crearía la Organización de las Naciones Unidas. La conformación de un bloque americano ante el sistema 
universal, como se verá más adelante, se consensuó bajo el entendido de que ciertos temas torales de política internacional, tales como la seguridad hemisférica, se reservarían para el ámbito regional.

Acorde con Morgenfeld, al interior del Departamento de Estado existían dos corrientes opuestas sobre el rumbo que debía tomar el "nuevo orden internacional". Por un lado, se encontraba la corriente "internacionalista", que pugnaba por la continuación de la estrecha relación con la Unión Soviética y la consolidación del Sistema de las Naciones Unidas sobre el Sistema Interamericano y, por el otro, la visión "regionalista", que buscaba la terminación de las relaciones con aquella y el establecimiento de un sólido andamiaje institucional a nivel regional con un grado suficiente de autonomía del sistema universal (Morgenfeld, 2010, p. 206). Al final, fue evidente la prevalencia de la visión regionalista.

Un ejemplo que evidencia la prevalencia de la visión regionalista fue el telegrama enviado por el secretario de Estado a los oficiales diplomáticos de Brasil, Chile, Costa Rica, Guatemala, Haití, México, Panamá, Uruguay y Venezuela, el 2 de enero de 1945, en el que se señaló la importancia de atender a la Conferencia de San Francisco con pleno entendimiento de los puntos de vista de los otros y con "mente abierta" para lograr una mayor efectividad en la cooperación para construir una estructura pacífica, con miras a la seguridad internacional. ${ }^{12}$

Es en este escenario que México ingresa con plenitud al nuevo sistema internacional, después de que anteriormente había tenido un reducido papel en los foros internacionales debido a represalias que impusieron los países occidentales a la revolución mexicana (Hernández, 2002, p. 403). En un breve recuento, en 1944 derivado de los acuerdos de Bretton Woods surgen el Fondo Monetario Internacional y el Banco Mundial, a través de las cuales se reorganizó el sistema financiero internacional, entre otras cosas, dando centralidad al dólar, y estableciendo una paridad fija entre el oro y esta moneda. Un año después, en 1945, se crea la Organización de las Naciones Unidas y se suscribe el Acuerdo General sobre Aranceles Aduanales y Comercio, lo que termina por configurar el nuevo escenario político y económico mundial. Posteriormente, en 1948, el Consejo Económico y Social de la Naciones Unidas (ECOSOC, por sus siglas en inglés) creó la CEPAL, para contribuir al desarrollo económico y social de la región que, como se verá más adelante, fue determinante para la cimentación teórica del nuevo modelo económico adoptado por los países latinoamericanos.

Por otra parte, gracias a un largo proceso de evolución que puede rastrearse hasta 1889 con la Primera Conferencia Internacional Americana y

\footnotetext{
${ }^{12}$ Foreign Relations of the United States (FRUS), Diplomatic Papers, 1945, General, The United Nations, vol. I.
} 
el movimiento del panamericanismo se consolidan las instituciones regionales de las Américas. En 1948 se establece la Organización de Estados Americanos y en 1959 el Banco Interamericano de Desarrollo.

En la Conferencia de Chapultepec (1945) se empezaba a perfilar lo que pasaría a ser este nuevo orden mundial que incluía a Estados Unidos como su centro principal. La primera de estas dos conferencias se celebró en el Castillo de Chapultepec, en la ciudad de México, del 21 de febrero al 8 de marzo de 1945, y se denominó Conferencia Interamericana sobre Problemas de la Guerra y de la Paz. Las resoluciones más importantes de esta conferencia estaban vinculadas al futuro del Sistema Interamericano y por lo menos tres resoluciones cobrarían gran importancia: una relativa al principio de solidaridad americana, que sería el germen de la asistencia recíproca frente a la agresión; otra relacionada con la creación de la organización regional permanente, y una más que propondría la vinculación del panamericanismo con el sistema general internacional, que nacería a partir de la Carta de San Francisco (Secretaría de Relaciones Exteriores, 2003). Siguiendo estas líneas, la Resolución VIII de la Conferencia Asistencia Recíproca y Solidaridad Interamericana derivaría en el Tratado Interamericano de Asistencia Recíproca, firmado en Río de Janeiro en 1947, y por su parte la Resolución IX de la Conferencia titulada Reorganización, Consolidación y Fortalecimiento del Sistema Interamericano tendría una influencia directa en el surgimiento de la Organización de Estados Americanos, que nace en la Novena Conferencia Panamericana celebrada en Bogotá en 1948 (Fagg, 1982, p. 218).

Dentro de la Conferencia de Chapultepec se constituyó la Comisión IV, que quedó dividida en las subcomisiones A y B. La Subcomisión B estaría encargada del planteamiento de problemas sociales, como la pobreza, y la búsqueda de su solución. Por su parte, la Subcomisión A sería la encargada del estudio y búsqueda de soluciones para los problemas económicos americanos. El primer problema que se planteó en la Subcomisión A fue el de determinar el alcance de los estudios y soluciones que podría abordar la conferencia, teniendo en cuenta que los planes económicos de posguerra serían considerados posteriormente en una reunión especial, como la Conferencia Técnico-Económica Interamericana programada en Washington. En este sentido, "quedó acordado que los delegados gubernamentales presentes en México deberían sentar tan solo las bases y orientaciones generales con respecto a la política americana de post-guerra, procurando no entrar en detalles técnicos que escaparan a sus posibilidades" (Ministerio de Relaciones Exteriores de Uruguay, 1946).

Cabe destacar que desde el inicio de las actividades y el planteamiento de los objetivos de la Subcomisión A, ya se observaban algunas posiciones encontradas entre la delegación de Estados Unidos y las delegaciones de 
los países latinoamericanos. Estos últimos se verían vulnerados si cesaban sus exportaciones para las exigencias de la guerra. Además, el desarrollo industrial latinoamericano, impulsado por el mismo conflicto bélico, fue otro factor que la mayoría de los países de la región debían de considerar en las soluciones que pudieran plantearse en la conferencia. No se deseaba perder la oportunidad de que América Latina abandonara su función pasiva de proveedora de materias primas y avanzara en su desarrollo económico. La región aspiraba a pasar a una era de industrialización -a la que, como se mencionó anteriormente, algunos países, como México, Argentina, Brasil y Chile ya habían pasado- que le permitiera atenuar la repetición de algunas crisis que había experimentado debido a su dependencia hacia los países desarrollados. Sin embargo, Estados Unidos ya había expresado claramente su desacuerdo con "la adopción de un sistema preferencial en los convenios comerciales entre los países de América [...] porque sus términos son incompatibles con la política tradicional de principios liberales de comercio internacional seguida por los Estados Unidos de América". ${ }^{3}$

Además, entre el 9 y 18 de mayo de 1944 se llevó a cabo en la ciudad de Nueva York la Conferencia de Comisiones de Fomento Interamericano. En ella se adoptaron "cuarenta y tres recomendaciones sobre diversos e importantes temas económicos, inspirados en principios liberales". ${ }^{14}$ Entre ellas, se planteó que para mejorar el estándar de vida de la población, los países de América Latina debían aprovechar las condiciones naturales existentes en los diversos países, y especializarse en la producción de aquellas materias primas que presentaran características económicas favorables. ${ }^{15}$ Esto se sustentaba en un principio económico liberal sobre el aprovechamiento de las ventajas comparativas en una economía. Es decir, la idea era que las economías de la región continuaran especializándose en la producción de bienes intensivos en el factor de producción abundante ${ }^{16}$ lo que mantendría a los países latinoamericanos, contra su voluntad, dentro del modelo de crecimiento hacia el exterior, surtiendo productos primarios a los países del centro.

En la Conferencia de Chapultepec participaron 20 países, todos los miembros de América, con la excepción de Argentina. La delegación estadunidense estaba compuesta, además, por representantes de grandes corporaciones en crecimiento deseosos de hacer negocios en el continente, lo cual se vio reflejado en la discusión de la conferencia. La delegación

\footnotetext{
${ }^{13}$ Conferencia Interamericana sobre Problemas de la Guerra y de la Paz, t. L-E-484, p. 5, Acervo Histórico Diplomático (en adelante AHD).

${ }^{14}$ Conferencia Interamericana sobre Problemas de la Guerra y de la Paz, t. L-E-484, p. 5, AHD.

${ }^{15}$ Conferencia Interamericana sobre Problemas de la Guerra y de la Paz, t. L-E-484, p. 6, AHD.

${ }^{16}$ A este principio se conoce hoy en día en la teoría económica como principio Heckscher-Ohlin.
} 
estadunidense renegaba de todo estatismo y lo consideraban un peligro para la libertad de empresa. Mientras la periferia abogaba por ideas claramente diferentes, trataban de revivir la idea de un Banco Interamericano y se resistían a la política arancelaria de Washington, lo cual les permitió moderar el avance librecambista durante Chapultepec (Rapoport y Spiguel, 2009, pp. 95-96).

La diferencia entre las metas económicas planteadas por Estados Unidos y las de los países latinoamericanos generó un ambiente de confusión y contradicciones en torno a la política económica que fuera más conveniente para las repúblicas ubicadas al sur de Estados Unidos. Las delegaciones de los países latinoamericanos consideraban que era "preciso meditar sobre las verdaderas posibilidades de América para intentar la transformación de la estructura de la economía mundial sobre bases liberales". ${ }^{17}$

La propuesta económica estadunidense de mayor significación, dentro de la Conferencia de Chapultepec, fue la llamada Carta Económica de las Américas. El proyecto se aprobó después de un acalorado debate donde los países latinoamericanos, pese a la resistencia de Estados Unidos, lograron realizar modificaciones fundamentales al texto original. La carta se dividió en tres partes: primero tiene una exposición de las premisas básicas del texto, en donde primordialmente se habla de las aspiraciones económicas de todo pueblo; en segundo lugar, la carta contiene una sección llamada Declaración de Objetivos, esta parte habla de las etapas por las que tendrían que pasar los Estados americanos entre el periodo de guerra, que aún continuaba, y el periodo de posguerra, pasando por una etapa de transición. Estas dos secciones no sufrieron modificaciones en la resolución final. Sin embargo, la tercera sección, denominada Declaración de Principios, fue modificada en varios puntos. Por ejemplo, Estados Unidos sugirió que el punto tres que se planteaba, se titulara Disminución de Barreras Comerciales, en el cual proponían atenuar los mecanismos proteccionistas de toda índole. ${ }^{18}$

Por su parte, la Comisión IV, además de sugerir el cambio de título del fragmento por Política Comercial Internacional, propuso que la disminución de barreras se diera "dentro de normas que aseguren a todos los pueblos de la tierra altos niveles de vida y desarrollo de sus economías sobre bases sólidas". ${ }^{19}$ Esta última frase iba en la dirección de los objetivos de industrialización de los países latinoamericanos. Es decir, una vez

\footnotetext{
${ }^{17}$ Conferencia Interamericana sobre Problemas de la Guerra y de la Paz, t. L-E-484, p. 6, AHD.

${ }^{18}$ Conferencia Interamericana sobre Problemas de la Guerra y de la Paz, t. L-E-487, pp. 222223, AHD.

${ }^{19}$ Conferencia Interamericana sobre Problemas de la Guerra y de la Paz, t. L-E-484, p. 18, AHD.
} 
que algunos sectores económicos en vías de desarrollo, como el industrial, se desarrollaran sobre bases sólidas, la propuesta de eliminar barreras de toda índole sería válida. ${ }^{20}$

Otro cambio importante en la Carta Económica tiene que ver con el punto cinco de la Declaración de Principios: la eliminación del nacionalismo económico. Para los representantes latinoamericanos, el texto debía hablar de la eliminación de los excesos de nacionalismo económico. Es decir, la Comisión IV hablaba de cooperación internacional y de evitar la excesiva restricción de las importaciones; mientras que Estados Unidos hablaba de eliminar el nacionalismo económico en todos sus aspectos, incluyendo la no restricción a las importaciones de productos manufacturados en América Latina. Sobre esta cuestión, la delegación de Brasil afirmaba que "[Había] pedido al señor Delegado de los Estados Unidos que debe considerarse el punto que nosotros tenemos a la vista, porque nosotros ya tenemos una industria bien desarrollada y lo fue bajo la tarifa aduanal. Si ahora aprobamos sin mayor detalle lo que se propone, podemos ocasionar un disturbio económico." ${ }^{21}$

Irónicamente, el delegado de Cuba mencionaba respecto a este punto que "nosotros estamos de acuerdo con lo que Estados Unidos propone, pero no quisiéramos hacerlo porque implicaría un suicidio". ${ }^{22}$ De la misma forma, la delegación de Uruguay expresó que "el abatimiento de las barreras arancelarias significaría para los países latinoamericanos una verdadera catástrofe" y, continúa, "se podrá producir ese abatimiento de barreras [...] sin que traiga como consecuencia una catástrofe, cuando se haya transformado la vida social del mundo". ${ }^{23}$ De esta forma, vemos que en su mayoría los países latinoamericanos más influyentes tenían la idea de continuar con un proceso de industrialización, que en algunos países como Brasil, México, Chile, Colombia, Perú y Argentina habían comenzado hacía más de una década y que en otros más pequeños surgió como producto del conflicto bélico. En ese sentido, autores como Villanueva afirman que la Conferencia de Chapultepec no implicó una ruptura con el modelo mercado internista que iniciaron ciertos países desde el comienzo del siglo XX (Villanueva, 1972, pp. 475-476).

\footnotetext{
${ }^{20}$ Las delegaciones que propusieron este último cambio en el texto de la carta fueron la cubana y la mexicana. Por su parte, Brasil también estuvo en desacuerdo con la propuesta estadunidense, porque el hecho de que el texto sólo contuviera la frase reducción de barreras de toda índole mataría la iniciativa particular de industrialización que su país ya tenía iniciada.

${ }^{21}$ Conferencia Interamericana sobre Problemas de la Guerra y de la Paz, t. L-E-466, p. 85, AHD.

${ }^{22}$ Conferencia Interamericana sobre Problemas de la Guerra y de la Paz, t. L-E-466, p. 85, AHD.

${ }^{23}$ Conferencia Interamericana sobre Problemas de la Guerra y de la Paz, t. L-E-466, pp. 8788 , AHD.
} 
Los puntos mencionados con anterioridad nos permiten mostrar claramente la posición que guardaba América Latina respecto a las tarifas arancelarias y desarrollo industrial. Esto contrasta con los objetivos estadunidenses de apertura comercial sin restricciones y del resurgimiento del modelo al que algunos autores llamarían de dependencia económica, en donde los países latinoamericanos volverían a su posición tradicional de exportadores de materias primas e importadores de manufacturas.

Finalmente, es importante mencionar el punto de la Carta Económica que está relacionado con la intervención gubernamental en la economía. Como mencionamos anteriormente, durante los años vigentes del modelo de sustitución de importaciones en los países de América Latina, los gobiernos desempeñaron un papel importante tanto en la aplicación de políticas que fomentaran el desarrollo industrial (exenciones fiscales, políticas arancelarias e inversiones en infraestructura pública) como directamente en el proceso productivo, donde realizó inversiones conjuntas con el capital nacional privado y extranjero, por ejemplo, en México, se invirtió en la industria eléctrica y en el sector siderúrgico. ${ }^{24}$ Estados Unidos, dentro de sus propuestas liberales expresadas en la Carta Económica, consideró que los países latinoamericanos debían abstenerse de "crear empresas oficiales que se dediquen al comercio". ${ }^{25}$ Finalmente se eliminó esta frase, incluida en el punto sobre la Inversión Privada e Intervención Gubernamental, y se dejó a discreción de los países de la región la decisión de emplear al gobierno como agente promotor del desarrollo económico, tanto en el sector de comercio como en la producción. ${ }^{26}$ Incluso a este punto se le cambió el nombre por "Inversión Privada" en el texto finalmente aprobado. Es decir, se eliminó el aspecto referente a la intervención gubernamental.

La redacción final de la carta se aprobó con las modificaciones hechas por la Comisión IV. ${ }^{27}$ Casi al término de la Conferencia, el 7 de marzo, también se aprobó la resolución complementaria L sobre Desarrollo In-

${ }^{24}$ Un estudio interesante que analiza algunas de las primeras inversiones conjuntas entre estos tres grupos es el de Mosk (1950).

${ }_{25}$ Conferencia Interamericana sobre Problemas de la Guerra y de la Paz, t. L-E-487, p. 223, AHD.

${ }^{26}$ Respecto al tema de la intervención gubernamental en la producción, Estados Unidos no fue tan estricto como en el caso del comercio. Sin embargo, los representantes latinoamericanos no permitieron restricciones en ninguno de los dos sectores mientras la intervención gubernamental fuera en beneficio del interés público. Véase Conferencia Interamericana sobre Problemas de la Guerra y de la Paz, t. L-E-466, p. 76-81, AHD.

${ }^{27}$ Esto gracias, en parte, a las declaraciones de William Clayton, delegado de Estados Unidos para los asuntos económicos en Chapultepec y subsecretario de Asuntos Económicos del Departamento de Estado, sobre "las proyecciones de las medidas de control de cambios y tarifas arancelarias, estableciendo categóricamente que serán aplicadas sobre una nueva realidad que se está estructurando". Estas declaraciones, de acuerdo con la delegación de Uruguay, "son tranquilizadoras para los países económicamente débiles y de industrialización incipiente" (Ministerio, 1946, pp. 169-170). 
dustrial, la cual exhortaba a los Estados americanos a impulsar la creación de nuevas ramas industriales, así como perfeccionar y ampliar las existentes, siempre y cuando las condiciones fueran favorables. También se recomendaba, para conseguir el financiamiento que esto requería, la rápida ratificación de los Convenios de Bretton Woods. Así, Estados Unidos se comprometía a facilitar maquinaria, equipo y medios de transporte para estimular la industrialización de los países latinoamericanos, a la vez que las inversiones extranjeras debían realizarse de modo tal que aseguraran al capital nacional una justa y adecuada participación en la formación y dirección de las empresas, procurando que estas no desplazaran a los capitales nacionales de las industrias, negocios y actividades ya existentes. Estas últimas eran claras concesiones de Estados Unidos para que los países latinoamericanos aprobaran la resolución (Morgenfeld, 2010, pp. 208-209).

El aparente éxito de los países de América Latina en las negociaciones de la Conferencia de Chapultepec, en buena medida, también se debió a que la región no figuraba como una de las prioridades de política exterior de Estados Unidos. Ante el rápido desarrollo del mundo bipolar, la atención política y económica de Washington se concentró, en primera instancia, en la reconstrucción de Europa occidental y en la contención de la influencia de China, principalmente en Japón y el sureste asiático. Ello facilitó que Washington cediera en temas centrales en la negociación de la Carta Económica de las Américas.

Sin embargo, la posición librecambista de Estados Unidos se volvió más radical en conferencias subsiguientes. Estados Unidos envió como delegado a Chapultepec al subsecretario de Asuntos Económicos del Departamento de Estado, William Clayton, y él mismo fue el ideólogo de la Carta de La Habana, a la que peyorativamente se le denominó el Plan Clayton. Esta carta se propuso en la Conferencia sobre el Comercio y Empleo, que inició en La Habana hacia fines de 1947, y "partía del supuesto de que la libre circulación de productos y capitales era la base del desarrollo económico. Por lo mismo, se recomendaba la reducción de tarifas y la paulatina eliminación de restricciones cuantitativas y de preferencias arancelarias, así como la supresión del control de cambios" (Torres, 1984, p. 296). Al igual que la Carta Económica de las Américas, la Carta de La Habana sufrió los ataques de distintos sectores de la economía mexicana, por ejemplo del sector obrero (Torres, 1984, p. 296). Además, para ese entonces las políticas proteccionistas en países como Argentina, Brasil, Chile y Colombia ya estaban bien determinadas (Bulmer-Thomas, 1994, p. 280). En el caso de México, desde mayo de 1944 se publicó un decreto en el Diario Oficial de la Federación (12 de mayo de 1944), en el cual se facultaba a la Secretaría de Hacienda a restringir o autorizar la importación de ciertos artículos. A partir de septiembre de 1945, la Secretaría de Hacienda 
comenzó a hacer un uso más frecuente de este decreto y restringió la importación de numerosos productos. De la misma forma, una circular del secretario de Hacienda de diciembre del mismo año confirmó la política proteccionista mexicana (Torres, 1979, pp. 191-192). Sin embargo, a pesar de que hubo confrontación, los países latinoamericanos se vieron en la necesidad de aceptar en gran medida el "Plan Clayton", ya que continuaban insistiendo para la obtención de ayuda económica por parte de Estados Unidos, por lo que ese elemento limitó sus reclamos.

\section{EL NACIONALISMO ECONÓMICO Y LA CEPAL}

Por su parte, el creciente consenso sobre la importancia de la industrialización en América Latina encontró soporte teórico e institucional en la CEPAL. ${ }^{28}$ Como se mencionó anteriormente, esta comisión fue creada en 1948 y, de acuerdo con Rosemary Thorp, tuvo que probarse a sí misma, en un periodo corto, si podría mantenerse viva (Thorp, 1998, p. 132). El grupo de jóvenes economistas, bajo el liderazgo de Raul Prebisch, tuvo que mostrar la existencia de un punto de vista latinoamericano válido para justificar la necesidad de generar una industria en los países de la región. En 1949 se creó la "tesis Prebisch", misma que fue modificándose a lo largo de los años cincuenta. Había tres justificaciones principales para la aplicación de un modelo de sustitución de importaciones en la región. En primer lugar, la restricción externa en el crecimiento económico era atribuida al deterioro de los términos de intercambio de productos primarios (precio) y a la carencia de acceso al mercado (volumen) para las exportaciones primarias, por lo que se requería alguna fuente doméstica de crecimiento. En segundo lugar, había una necesidad social de que se incrementara el empleo rápidamente para absorber la creciente fuerza de trabajo y ofrecer mejores oportunidades para los desempleados del sector de la agricultura tradicional, que los sectores relacionados con bienes primarios no podían lograr. En tercer lugar, la única manera de generar un rápido progreso tecnológico era por medio de la industrialización, ya que los beneficios de cualquier progreso en la productividad de las exporta-

\footnotetext{
${ }^{28}$ Mientras que la Teoría de la Dependencia fue un gran desarrollo teórico desde la periferia para pensar el desarrollo desde una mirada compleja, el nuevo paradigma tenía bases vulnerables. De acuerdo con Aldo Ferrer (2005), este no sobrevivió las limitaciones emergentes del Estado desarrollista coexistiendo con las mismas raíces del privilegio y la concentración de la riqueza del pasado histórico. Cuando el poder céntrico se reconstituyó en torno de la hegemonía norteamericana y las nuevas fuerzas de la globalización operantes en los mercados financieros, las corporaciones trasnacionales y las comunicaciones, otra vez, el pensamiento hegemónico en América Latina pasó a ser formulado de los centros y, por las mismas causas, fundadas en la debilidad de la densidad nacional de los países latinoamericanos.
} 
ciones de bienes primarios se acumulaban en los países importadores más que en los exportadores (Fitzgerald, 2000, pp. 60-61).

De igual manera, en los trabajos desarrollados por Diamand (1972) y Braun y Joy (1968), se vio plasmada la dinámica macroeconómica (latinoamericana en general, aunque de manera más marcada en el caso argentino) de las décadas de los años cincuenta y sesenta, reflejada en los modelos de stop and go, los cuales representaban la realidad de una economía cuyas exportaciones (principalmente agropecuarias) se vieron estancadas, mientras que el estado de incipiente desarrollo industrial determinaba que el crecimiento sostenido del PIB demandara de la importación cada vez más bienes de capital e insumos, así como de la adquisición de combustibles y manufacturas que no se producían al interior de los países en vías de desarrollo.

Prebisch argumentaba que en estas nuevas condiciones mundiales, que además implicaron el cambio de centro global, del Reino Unido a Estados Unidos, América Latina debía adaptarse y encontrar nuevas reglas concordantes con la realidad, y mientras eso no ocurriera continuaría una tendencia al desequilibrio, puesto que mientras Estados Unidos disminuía el coeficiente de importaciones, en los países latinoamericanos tendió a subir el coeficiente de importaciones en dólares, lo que les obligó a tomar acciones de defensa para atenuar sus efectos (Prebisch, 1949, p. 23).

De igual manera, existía la idea de que durante el periodo del capitalismo liberal del primer tercio del siglo XX, las economías latinoamericanas no obtuvieron utilidades económicas de manera directa de los sectores controlados por intereses extranjeros, incluida la producción de productos primarios cuando esta era realizada por agentes foráneos. Es decir, se pensaba que los sectores manejados por los extranjeros eran enclaves, puesto que la mayoría de sus beneficios salían del país. ${ }^{29}$ Prebisch alegaba que el

\footnotetext{
${ }^{29}$ Esta noción de enclave ha sido desmentida en algunos estudios, como el de Kuntz (2007, pp. 406-444) sobre el comercio exterior en México en la era del capitalismo liberal. En este estudio, la autora analiza en detalle los productos mexicanos de mayor exportación entre 1870 y 1929. Su enfoque está en determinar los enlaces, externalidades positivas y derramas salariales y fiscales que estos productos tienen sobre la economía mexicana para no poderse considerar en ninguno de los casos enclaves. Se encuentra que la actividad henequenera estuvo casi completamente en manos de mexicanos, lo que tenía un alto valor de retorno para la economía mexicana. En el caso de la minería, Kuntz afirma que aunque el auge minero estuvo protagonizado por el capital extranjero, la derrama salarial de esta actividad fue muy importante. Según sus estimaciones, esta derrama pudo haber sido de aproximadamente $27 \%$ del valor de la producción minera del país para 1907. El sector minero también tuvo una importante derrama fiscal, por medio de los impuestos, además de que los metales mexicanos tenían un alto grado de procesamiento industrial dentro del país, que se incrementaba constantemente y que generaba una serie de externalidades positivas. Sobre el petróleo, Kuntz afirma que aunque las exportaciones relacionadas con este hidrocarburo comenzaron hasta 1911 y que las empresas que lo manejaban eran extranjeras, la contribución del sector petrolero a la economía mexicana aumentó durante la década de 1920 en virtud de que las plantas de refinación hicieron que el componente primario de las exportaciones
} 
subdesarrollo latinoamericano era el resultado de la posición latinoamericana en la economía mundial, y de la adopción de las políticas económicas liberales capitalistas. Su teoría era una suerte de continuación de la tradición antiimperialista y antimarxista, pero de una forma más sutil, sustituyendo, por ejemplo, términos de imperialismo más precisos y políticamente fuertes, por la expresión "relaciones centro-periferia" (O’Brien, 1974, p. 9).

Celso Furtado y Helio Jaguaribe, entre otros, enriquecieron la teoría, al contrastar las causas exógenas, derivadas del comportamiento del orden mundial, con las endógenas, resultantes de la propia realidad de los países latinoamericanos. El subdesarrollo latinoamericano resultaba así de complejas relaciones entre los intereses dominantes en el orden mundial y las elites domésticas, beneficiarias del modelo e incapaces de conducir a sus países por el sendero del desarrollo económico y social. La teoría de la dependencia fue un importante subproducto del estructuralismo latinoamericano (Ferrer, 2005).

La CEPAL argumentaba que la aplicación del nuevo modelo requería de la promoción deliberada de la industrialización por parte del gobierno, ya que había una serie de deficiencias en infraestructura e institucionales que no permitirían a las economías latinoamericanas responder a la señal del cambio en los precios de manera automática (Thorp, 1998, p. 133). Además proponía que América Latina necesitaba industrializarse detrás de altas barreras restrictivas, y que estas no sólo ayudarían a las "industrias nacientes", sino también, dado el desempleo estructural y la infrautilización del capital, ayudaría a la asignación óptima de los recursos. Posteriormente, la CEPAL añadió la necesidad de crear un Mercado Común Latinoamericano para alcanzar economías de escala. De esta manera la región latinoamericana lograría un camino de desarrollo orientado hacia adentro (O’Brien, 1974, pp. 9-10).

El crecimiento de la industria generaría una independencia de las inestables y poco dinámicas exportaciones primarias. Los flujos de capital extranjero eran vistos como positivos para lograr los objetivos de industrialización, pero la CEPAL ${ }^{30}$ de los años cincuenta previó que estos flujos vendrían principalmente en forma de préstamos al sector público. Sin em-

petroleras se redujera paulatinamente, además de que una parte importante y creciente de la producción del petróleo en México permaneció en este país para su consumo.

${ }^{30}$ Mientras que Prebisch estaba a favor de la industrialización de la periferia, una de las primeras críticas a la política de industrialización por sustitución de importaciones se presentó desde las filas de la misma CEPAL. El manifiesto de la CEPAL ya había reflejado recelos acerca de la industrialización latinoamericana, posteriormente, alrededor de la década de los años sesenta publicó una serie de críticas al proceso de industrialización. Los estructuralistas criticaron el proceso "realmente existente" de sustitución de importaciones como concentrador y excluyente ya que los frutos del avance tecnológico traído por la industrialización se concentraban en las manos de los 
bargo, este dinero, que se pensaba vendría de Estados Unidos, no llegaría, ya que, como se mencionó anteriormente, este país se comprometió con la reconstrucción de la Europa de la posguerra. Asimismo, Estados Unidos comenzó a considerar los problemas económicos de la región como esencialmente internos y mostraron más interés en la lucha anticomunista que por el desarrollo económico latinoamericano. Una versión del Plan Marshall de Europa fue propuesto para América Latina en la Conferencia Interamericana de Bogotá de 1948, pero sin éxito, lo que hizo que el modelo de industrialización latinoamericano finalmente dependiera de la inversión extranjera directa (Cosío, 1964). De tal suerte que, pese a que Estados Unidos se negó a brindar asistencia económica para la industrialización de los países de la región, el modelo cepalista no implicó ningún obstáculo al creciente aumento de inversión extranjera directa proveniente de dicho país, sobre todo en aquellos Estados latinoamericanos en los que se permitía el acceso irrestricto del capital privado internacional en áreas centrales de la economía. Al mismo tiempo, el aumento de las barreras proteccionistas estadunidenses, después de la Conferencia de La Habana en 1947, también contribuyó a que la industrialización de América Latina fuera orientada hacia el mercado interno (Fitzgerald, 1994). ${ }^{31}$

Miguel Alemán, en línea con lo que la CEPAL apoyaba, llegó a la presidencia de México en 1946, con un programa económico que hizo hincapié en la producción industrial mediante el apoyo decidido del Estado y el fomento a la inversión privada internacional para el desarrollo de sectores productivos. Entre las medidas aplicadas para alentar y proteger a la industria se contó con el alza de aranceles y con la puesta en marcha de un mecanismo de licencias previas para las importaciones, que agregó otro instrumento al Estado para regular la economía nacional. También se encauzó un mayor volumen de crédito a la industria y se reestructuró el régimen de exenciones fiscales para las mismas. En 1952, aproximadamente la mitad de los créditos otorgados por la banca privada se destinaron a actividades industriales (Medina, 1995, p. 136). NAFINSA continuó con su promoción de la industrialización, ahora concentrándose más en proyectos de infraestructura.

El patrón seguido por Alemán fue común en el grueso de los países latinoamericanos; la mayoría de ellos fueron influenciados por la CEPAL y justificaron sus medidas económicas promotoras del desarrollo industrial con el pensamiento cepalista durante las siguientes décadas. La estrategia

dueños del capital, excluyendo a la mayoría y agravando las desigualdades en la distribución del ingreso (Kay, 1991, p. 105).

${ }^{31}$ Originalmente, se pensaba que la expansión del sector manufacturero se basaría en el mercado interno, pero que eventualmente las manufacturas intensivas en el factor trabajo, como textiles, podrían ser exportadas. 
proteccionista básicamente se basó en una combinación de altas tarifas en bienes de consumo, la exención de tarifas en muchos bienes de capital e intermedios, y un tipo de cambio sobrevaluado, lo que en algunos de los casos significaba que los bienes no sujetos a tarifas fueron extremadamente baratos (Thorp, 1998, p. 137). Todo lo anterior apoyado por un Estado fuertemente involucrado en el desarrollo industrial. Se fueron creando así las bases para que la Organización de Estados Americanos aprobara formalmente la Operación Panamericana en marzo de 1959, para el despliegue del discurso desarrollista de la CEPAL y la resultante fundación de la Asociación Latinoamericana de Libre Comercio en febrero de 1960 (Suárez y García, 2008, pp. 104-105).

\section{CONClusiones}

En este artículo se ha intentado demostrar que las propuestas económicas hechas por Estados Unidos en la Conferencia de Chapultepec de 1945, en especial en la Carta Económica de las Américas, no lograron frenar el avance de las políticas de industrialización que los países latinoamericanos iniciaron años atrás. Las propuestas estadunidenses fueron básicamente liberales e incluían la eliminación de barreras comerciales de toda índole, para que de esta manera se pudiera continuar con el modelo de desarrollo económico que había caracterizado a América Latina después del último tercio del siglo XIX y principios del XX. Es decir, se pretendía que Latinoamérica continuara siendo proveedora principalmente de productos primarios para los países industrializados. Sin embargo, como se mostró en el trabajo, una importante consecuencia de la Gran Depresión de 1929 fue la aceleración del proceso de sustitución de importaciones y la industrialización doméstica de gran parte de los países de América Latina, proceso fortalecido por la segunda guerra mundial. De esta forma, la postura de los países al sur de Estados Unidos fue de moderar el avance de la apertura comercial tan violenta que sugería el vecino del norte. No obstante, los países latinoamericanos no adoptaron una postura de radical oposición a las políticas librecambistas de Estados Unidos, debido a que mantenían la expectativa de recibir ayuda económica por parte de dicho país. Se buscaba que se implementara una suerte de Plan Marshall para la región latinoamericana, que aunque finalmente no se llevó a cabo, logró moderar de manera considerable la postura de los países de la región. Esto se puede observar claramente en el texto final que se aprobó de la Carta Económica de las Américas, que sufrió varios cambios respecto al texto original propuesto por Estados Unidos, después de un intenso debate en el marco de la Conferencia de Chapultepec. 
En años subsiguientes, la postura estadunidense se intensificó, lo que fue evidente en la Carta de La Habana, propuesta en la Conferencia de La Habana de 1947, así como en la suscripción del Tratado Interamericano de Asistencia Recíproca en el mismo año. Sin embargo, los países latinoamericanos lograron moderar la implementación de las políticas librecambistas impulsadas por Estados Unidos y mantuvieron altas barreras arancelarias para evitar un efecto negativo sobre su industria manufacturera. Además, la política de industrialización de la región encontraría justificación teórica en los trabajos de la CEPAL de finales de los años de 1940, lo que fortaleció en la práctica la implementación del modelo económico nacionalista en la región. De esta manera, los países latinoamericanos continuaron con el modelo de sustitución de importaciones por muchos años más, hasta que finalmente, a partir de la revolución cubana en 1959, se radicalizó la postura económica y política estadunidense hacia Latinoamérica, lo que influyó sustancialmente en la debacle económica de dicho modelo, cuyo fin fue estar en crisis en la década de los años setenta del siglo XX.

\section{LISTA DE REFERENCIAS}

Braun, O. y Joy, L. (1968). A model of economic stagnation: A case study of the Argentine economy. The Economic Journal, 78(312), 868-887. DOI: 10.2307/2229183

Bulmer-Thomas, V. (1994). The economic history of Latin America since independence. Cambridge: Cambridge University Press.

Bulmer-Thomas, V. (1997). Las economías latinoamericanas, 1929-1939. Historia de América Latina, 11, 3-46.

CÁrdenas, E. (1982). Mexico's industrialization during the Great Depression: Public policy and private response (Tesis doctoral). Universidad de Yale, Estados Unidos.

Cárdenas, E., OcAmpo, J. A. y ThOrp, R. (eds.) (2000). An economic history of twentiethcentury Latin America. Hampshire: Palgrave.

Cosío, D. (1964). American extremes. Austin: University of Texas Press.

Diamand, M. (1972). La estructura productiva desequilibrada argentina y el tipo de cambio. Desarrollo Económico, 12(45), 25-47. DOI: 10.2307/3465991

FAGG, J. E. (1982). Pan Americanism: Its meaning and history. Florida: Robert E. Krieger Publishing Company.

Ferrer, A. (2005). El rotundo triunfo del estructuralismo latinoamericano. Buenos Aires: Universidad de Buenos Aires.

Fishlow, A. (1972). Origins and consequences of import substitution in Brazil. En L. Dimarco (ed.), International economics and development: Essays in honor of Raúl Prebisch (pp. 311-365). Nueva York: Academic Press. 
FitzGerald, E. V. K. (1994). ECLA and the formation of Latin American economic doctrine in the 1940's. En D. RocK (ed.), Latin America in the 1940's: War and postwar transitions (pp. 89-108). Berkeley: University of California Press.

FitzGERALD, E. V. K. (2000). ECLA and the theory of import-substituting industrialization in Latin America. En E. CÁrdenas, J. Ocampo y R. ThorP (eds.), An economic history of twentieth century Latin America. Vol. 3: Industrialization and the State in Latin America. Basingstoke: Palgrave.

Galindo, J. (2012). Some considerations regarding the persistence of the economic elite in Mexico in the first half of the twentieth century. Bulletin of Latin American Research, 32(2), 149-162. DOI: 10.1111/j.1470-9856.2012.00769.x

HABER, S. (1989). Industry and underdevelopment: The industrialization of Mexico, 18901940. Stanford: Stanford University Press.

Hernández, A. (2002). México: una breve historia. México: Fondo de Cultura Económica.

KAY, C. (1991). Teorías latinoamericanas del desarrollo. Nueva Sociedad, 113, 101-113.

Knight, A. (1991). Rise and fall of cardenismo. En L. Bethell (ed.), Mexico since independence (pp. 241-320). Nueva York: Cambridge University Press.

Kuntz, S. (2007). El comercio exterior de México en la era del capitalismo liberal, 1870-1929. México: El Colegio de México.

Medina, L. (1995). Hacia el nuevo Estado mexicano, 1920-1994. México: Fondo de Cultura Económica.

Millán, H. (1998). Neoliberalismo y transición en México. México: El Colegio Mexiquense.

Ministerio de Relaciones Exteriores de Uruguay (1946). Conferencia Interamericana sobre Problemas de la Guerra y de la Paz. Montevideo: Sección Prensa, Informaciones y Publicaciones.

Mosk, S. (1950). Industrial revolution in Mexico. Berkeley: University of California Press.

Morgenfeld, L. A. (2010). Argentina y la vuelta al sistema interamericano: el largo camino a Chapultepec. Relaciones Internacionales, 19(39).

North, D. (1993). Instituciones, cambio institucional y desempeño económico. México: Fondo de Cultura Económica.

O'Brien, P. (1974). A critique of Latin American studies (Occasional papers, 12). Londres: Institute of Latin American Studies.

Prebisch, R. (1949). El desarrollo económico de la América Latina y algunos de sus principales problemas. Naciones Unidas: Comisión Económica para América Latina y el Caribe.

Rapoport, M. y Spiguel, C. (2009). Relaciones tumultuosas. Buenos Aires. Plus Ultra.

Secretaría de Relaciones Exteriores (2003). Conferencia Interamericana sobre Problemas de la Guerra y de la Paz. México: Autor.

SuÁrez, L. y GarcíA, T. (2008). Las relaciones interamericanas. Continuidades y cambios. Buenos Aires: Consejo Latinoamericano de Ciencias Sociales.

Tannenbaum, F. (1956). México: The struggle for peace and bread. Nueva York: Alfred A. Knopf. 
ThORP, R. (1998). Progress, poverty and exclusion, an economic history of Latin America in the twentieth century. Maryland: John Hopkins University Press.

Thorp, R. y LONDOÑO, C. (2000). The effect of the Great Depression on the economies of Peru and Colombia. En J. OcAmpo, E. CÁrdenas y R. Thorp (eds.), An economic history of twentieth-century Latin America. Vol. 2: Latin America in the 1930s. The role of the periphery in world crisis. Londres: Palgrave Macmillan

Torres, B. (1979). México en la segunda guerra mundial. Historia de la revolución mexicana, 1940-1952 (t. 19). México: El Colegio de México.

Torres, B. (1984). Hacia la utopía industrial. Historia de la revolución mexicana, 1940-1952

(t. 21). México: El Colegio de México.

Villanueva, J. (1972). El origen de la industrialización argentina. Instituto de Desarrollo Económico y Social, 12(47), 451-476. DOI: 10.2307/3466076

\section{OTRAS FUENTES}

\section{Archivos}

AHD Acervo Histórico Diplomático.

FRUS Foreign Relations of the United States, The United Nations.

\section{Hemerografia}

Diario Oficial de la Federación, México. 